\title{
0446 SUPPORT MECHANISMS AMONG OLDER PATIENTS - A QUALITATIVE STUDY FROM PAKISTAN
}

S M Khan*, L Laflamme Correspondence: Department of Public Health Sciences, Karolinska Institutet, Division of Global Health/IHCAR, Nobels vag 9, Karoliksa Institutet, Stockholm, SE 171 77, Sweden

\subsection{6/ip.2010.029215.446}

As ageing is an upcoming issue in low-income countries, an increase dependence on familial support is expected. Yet, traditional familial support mechanisms are threatened by declining family size, urban migration and globalisation. Our aim for this qualitative study was to explore the personal experiences and perception of elderly Pakistani patients about social and family support dynamics. The study was nested in a larger quantitative study. Patients attending the outpatient department of a tertiary care hospital were interviewed. Content analysis of data resulted in identification of one main theme, "Ageing exposes limitations in the support systems to address disability, reliance and vulnerabilities" and two sub-themes, (1) Disability and Vulnerability; (2) Support and Expectation.

The driving forces behind limitation in the support systems were functional disability, illness, inability to pay for healthcare, and disobedience and expectations from children. In addition, due to lack of old age social benefit systems elderly became dependent on their children for their physical and emotional care. They perceived old age as a tough stage of life, and felt themselves as a financial burden on their children. The elderly cited the concern regarding deteriorating social support system due to a crumbling value system of the society. Further, looking after the elderly was a value and responsibility of the children, society and government. Most of the participants refused to avail alternative accommodation like old homes, as they wanted to live with their loved ones in the current setup even with all difficulties. Strengthening of systems could enable elderly to cope. 